\title{
Un nouvel agent pour faciliter l'absorption des graisses
}

L'absorption défectueuse des graisses est un des phénomènes essentiels en pathologie de Гintestin grêle: de la sprue spectaculaire aux discretes iléites chroniques, en passant par 1'insuffisance pan-créatique et les courts-circuits resultant des operations sur Гestomac, on retrouve constamment un certain degré de stéatorrhée. Cette «indigestion»n'est pas seulement le témoin d'une alteration jéjuno-iléale, fonctionnelle ou organique, mais a pour consequence Tamaigris-sement consécutif à la perte des aliments les plus riches en calories.

Les moyens thérapeutiques utilises jusqu'ici pour combattre les stéatorrhées restent assez primitifs: la restriction des corps gras dans la nourriture constitue le plus simple et le moins satisfaisant, par ses consequences sur la nutrition de Гorganisme. L'administration de ferments divers n'a pas donné les résultats escomptés, peut-être par suite d'une posologie trop timorée. L'acide folique a fait avancer le problème du traitement des sprues, mais non des autres formes d'iléiie.

Des chercheurs de la Harvard Medical School de Boston nous proposent une autre solution, en s'adressant à des substances à pouvoír mouillant, c'est-à-dire capables d'abaisser la tension super-ficielle. C. M. Jones, P. J. Culver, G. D. Drummey et A. E. Ryan (Annals of internal Medicine, vol. 29, p. 1-10, juillet 1948), ont choisi dans ce vaste groupe chimique, le derive poly-oxy-éthylénique du mono-oléate de sorbite (specialise par Abbott sous le nom de Sorlate, en capsules de 0,5 g.), et se sont d'abord assures de son inocuité sur Гanimal, pendant plusieurs generations, puis sur Thomme, à très forte dose (15 g. par jour), pendant des mois.

Ils ont procédé ensuite à trois ordres de recherches sur le plan cliniques:

Courbes de la vitamine A dans le serum après ingestion de 200000 unites, additionnées ou non de 2 g. de Sorlate: celui-ci augmente Tabsorption de 400 à $500 \%$.

Dosages des lipides dans les selles de quatre gastrectomies subtotales, en comparant $\Gamma$ efFet du Sorlate à celui des acides déhydro-

Referate - Abstracts - Analyses

39

cholique, folique ou chlorhydrique, des extraits pancréatiqae ou hépatique: ces derniers ne modifiaient pas le contenu fécal, alors que le premier permettait la consommation de doses énormes de corps gras (jusqu'à $183 \mathrm{~g}$. par jour), ou ramenait $\Gamma$ absorption défec-tueuse à des limites normales.

c) Une malade atteinte de sprue, traitée par le regime et $\Gamma$ acide folique, avait vu ses principaux symptômes s'amender, mais Гamai-grissement et la stéatorrhée persistaient (perte de $22 \mathrm{~g}$. de graisses par jour). Avec le Sorlate, la malade engraissa de $10 \mathrm{~kg}$., et $\Gamma$ ex-crétion fécale tomba de 17 à $5,4 \%$.

Culver et ses collaborateurs admettent que le poly-oxy-éthylène du mono-oléate de sorbite agit en modifiant le pouvoir superficiel des particules graisseuses de façon à favoriser leur emulsion et leur dispersion. La posologie de choix consiste en 2 g., trois fois par jour, pour une ration de 2500 à 3000 calories avec au moins 125 à $150 \mathrm{~g}$. de graisse; le traitement doit être poursuivi plusieurs semaines ou même plusieurs mois pour en tirer tout le benefice desirable. 
M. D.

Referate - Abstracts - Analyses

Bearbeitet von: - Edité par:

F. Astraldi, Roma; M. Demoîe, Geneve; H. E. Edwards, London; L. v. Friedrich, Budapest; K.

Herfort,

Praha; Hulst, Utrecht; Th. Hunt, London; H. Jenzer, Bern; H. Kapp, Basel; N. Lloret, Barcelona. Alle Arbeiten der unten aufgeführten Zeítschriften werden in der Literaturübersicht referiert; Dans cette rubri $<\uparrow$ ue seront analyses tous les articles originaux paras dès 1948 danskles péríodiques suivants:

Acta Gast $\Gamma o e n t e r o l o g i c a ~ B e l g i c a \quad$ Arch, españ. e $\pi$ ferm. apar digest.

American Journal of Digestive Diseases Gastroenterology (Baltimore)

Archives des maladies de Vappareil digestif Review of Gastroenterology

Arch. ital. malat. apar. digest. Gastro-Enterologia Bohema

VII. Dünndarm

(Fortsetzung - Continuation)

4. Jejunitis necroticans Fick, A.K. et Wolken, A. P.: Necrotic jejunitis. Lancet 256, 519 (1949).

Durant ces dernières années, on put observer en Allemagne du Nord une épidémie de maladie grave de $\Gamma$ intestin caractérisée par les signes suivants: après un stade prodromique court et inconstant, les malades sont pris de violentes douleurs loca-lisées un peu au-dessus de Гombilic et à gauche de la ligne médiane. L'abdomen est tendu, resistant, le patient nauséeux. L'êtat general est mauvais, on constate souvent un collapsus circulatoire, la leucocytose monte à 16000 à 18000 et la mort

40

Referate - Abstracts - Analyses

peut survenir dans les 24 heures. Les malades qui passent cette période, souffrent alors de diarrhées sanguinolantes, et d'obstruction intestinale du type de Гileus paralytique. La temperature voisine 38 , la sedimentation est accélérée, les urines sont normales ainsi que Гexamen du sue gastrique.

Le diagnostic est difficile à faire, la radiologie montre des images de rigidité des parois jéjunales, avec une forte distension gazeuse. On confond surtout avec une perforation d'estomac, une obstruction du grêle, ou une pancréatite aiguë. La laparotomie ou la nécropsie révèlent une grave inflammation et une necrose d'une plus ou moins grande partie de jejunum. La paroi est rigide, fortement ædématiée, rétrécissant la lurnière de Гintestin ce qui est, avec arrêt du péristaltisme, une des causes de Tobstruction. La necrose progresse sous forme d'ulcération atteignant tout à tour toutes les couches de la paroi intestinale et se terminant par une perforation ou par une hémorragie parfois fatale. L'histologie montre aussi une necrose qui debute dans la muqueuse, puis atteint la sous-muqueuse qui s'ædématie, et devient le siege d'hémorragies. Une infiltration leucocytaire plus ou moins impor-tante justifie le terme de «jéjunite» donné à la maladie. Les auteurs considèrent ce syndrome comme une maladie nouvelle, n'ayant aucun rapport avec les lesions connues de la paroi intestinale, en particulier avec les inflammations purulentes. L'étiologie precise est inconnue, cependant, on peut admettre qu'il s'agit d'une maladie infectieuse spéecifique à laquelle prédisposent les fortes restrictions dont souffrent encore les populations allemandes. Zeissler aurait isolé un microorganisme spécifique, du groupe des clostridium, le Cloente-rotoxicum qui jouerait un role important dans la jéjunite nécrosante. Les cas les plus sévères relèvent de la chirurgie (exclusion, resection, fistule sur Гintestin grêle), tandis que les cas plus bénins furent traités par les sulfamidés, et le traitement antichoc classique. 
Prévot A.R.: Une nouvelle maladie huniaine à anaérobie : l'entérite nécrosante de Hambourg. Sem. Hop. Paris; 25, 1637-1639 (22 mai 1949).

En 1946 éclata à Hambourg une maladie foudroyante, caractérisée par deux à trois jours d'entérite aiguë, terminée par la mort (parfois après 12 heures seulement), due à une necrose de l'intestin, accompagnée d'ædèmes sanglants. Jeckeln baptisa la nouvelle affection «enteritis necroticans».

Son étiologie fut reconnue en 1948 par Zeissler et Rasfeld (voir Brit. med. J., 12 févr. 1949), qui isolèrent le clostridium welchíi type F ou Welchía agni, var. homojiitoxicus, microbe anaérobie dont $P$. décrit les caractères distinctifs.

Cette découverte se traduisit par une possibilité thérapeutique: sérothérapie spécifique ou polyvalente antigangréneuse combinée aux sulfamidés et au Marbadal ou Marfanil.

5. Tuberculosis

Esteban, J. M. Tuberculosis del intestine delgrado (Tuberculose de l'intestin grêle). Rev. Clin. Españ.; 28, 166-171 (1948)

Selon les experiences de l'auteur, chez tout tuberculeux pulmonaire actif ou non, bacillifère au non, avec ou sans troubles de Гappareil digestif, on doit réaliser une exploration systématique de ce système. E. trouve plus de $77 \%$ de tuberculose

Referate - Abstracts - Analyses

41

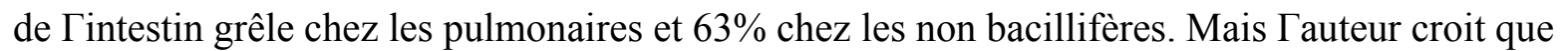
ce pourcentage est encore plus élevé. On doit répéter Гexamen de lïn-testin grêle chaque fois que Ton observe des alterations dans la lesion pulmonaire.

Mason, E. F., Gi idelbaugh, W. W. et Ward, M.: Streptomycin in the treatment of tuberculous enteritis. Am. J. med. Sci.; 217, 28-46 (1949).

II s'agit de cas des hôpitaux de l'armée américaine, présentant line tuberculose intestinale secondaire à une lesion pulmonaire et traités par la streptomycine. Le diagnostic intestinal fut clinique et radiologique, mais sans preuve bactériologique on anatomique. Le debut des symptômes abdominaux remontait à 4 à 6 mois avant Гinstauration du traitement; diarrhées, douleurs, nausées et vomissements, ballon-nement, parfois du sang et du pus dans les selles, perte de Гappétit et mauvais état general.

La streptomycine fut administrée à la dose de 1 à 2 grammes par jour durant 60 à 120 jours, en injections intramusculaires. Per os, Faction est aussi bonne, mais se fait attendre quatre à six semaines. Les résultats cliniques furent excellents: en une semaine, les signes abdonimaux disparaissent, les douleurs cèdent parfois en 24 à 48 heures, les autres symptômes disparaissent, la diarrhée diminue, Гappétit revient rapidement. La courbe du poids commence à remonter dès le debut du traitement.

La tuberculose pulmonaire, par contre, n'est que peu influencée, et 8 cas seule-ment sur 33 virent les $\mathrm{B} \mathrm{K}$ disparaître de leurs crachats après la guérison des lesions intestinales.

Les documents radiologiques de 17 cas montrent une guérison complete dans 6 cas, une persistance de quelques images anormales dans 8 cas sans grands change-ments malgré Tamelioration clinique évidente. L'on ne peut attendre un grand changement de $\Gamma$ image radiologique dans les cas où il y a une ulcération avec blocage lymphatique.

Markoff, N.: Die Streptomycinbchandlung der Darmtuberkulose. Schweiz. med. Wschr.; 78, 329-332 (1948).

Über die Streptomycinbehandlung der Darmtuberkulose liegen bis jetzt nur spärliche Angaben vor. Der Verf. berichtet in Form einer vorläufigen Mitteilung über seine eigenen Erfahrungen an 
fünf Fallen. Das Streptomycin wird parenteral und vor allem enteral in Form von hohen Einläufen gegeben $(250 \mathrm{mg}$ in $500 \mathrm{ccm}$ physiologischer Kochsalzlösung, der einige Tropfen Opiumtinktur beigefügt werden). Toxische Nebenerscheinungen wurden keine beobachtet, da die Resorption aus dem Darm gering zu sein scheint; außerdem waren die verwendeten Streptomycin -mengen relativ klein (in einem näher beschriebenen Fall 40,7 g in 48 Tagen). Die Behandlungsresultate waren außerordentlich günstig. Es konnte vorerst schon nach kurzer Zeit eine deutliche Entgiftung festgestellt werden, dann kam es zuJ Regulierung der Darmfunktion und Normalisierung des Stuhles. Die Geschwürs-heilung konnte röntgenologisch festgestellt werden, ohne daß es in der vorläufig noch kurzen Beobachtungszeit zu einer vollständigen Rückbildung der Darmverän-derungen gekommen ware. Die Resultate sind auch bei hoffnungslosen Fallen günstig. Hervorgehoben sei, daß die hier beschriebenen Beobachtungen sich nur auf die sekundäre Enterocolitis tuberculosa beziehen; über die Behandlung der primären Darmtuberkulose bestehen noch keine Erfahrungen.

42

Referate - Abstracts - Analyses

Silvani, H. L., Rothenberg, S., Warmer, H., Amluxen, J., McCorkle, H. J. Experiences de laboratoire et experiences cliniques avec la streptomycine dans le traitement d'infections d'origine intestinale. Surg. Gyn. Obst.; 85 (1947).

Un groupe de 13 malades atteints d'infections intraabdominales graves, furent traités par injection de streptomycine. Le résultat du traitement par la streptomycine semble avoir été utile dans un cas, peut-être utile dans un autre, douteux dans les deux suivants, très douteux dans cinq autres et nul dans les quatre derniers.

Dans une série d'animaux chez lesquels on a provoqué une pêritonite diffuse brutale d'origine appendiculaire, $\Gamma$ emploi de la streptomycine en usage local et general semble avoir prolongé la vie des animaux d'expérience, mais n'a pas eu d'effet notable sur la flore bactérienne du liquide peritoneal et n'a pas réussi à sauver la vie des animaux.

6. Lambliasis

Szílard, Z.; Les problèmes dans les maladies lambliaßiques. Arch. Mai. App. Dig.; 37, 354-357 (1948).

Court expose des inconnues dans la pathogénie des lambliases; pour S. la contradiction entre le nombre de personnes infectées et le peu de malades qui en souffre s'explique par une sensibilisation ou des stigmates neuro-vêgétatifs, mais il ne nous apporte aucun argument probant personnel à $\Gamma$ appui de sa these.

Hutet, M. G.: Traitement de la lambliase par l'association Quinacrine-Stovarsol alternés. Arch. Mai. App. Dig.; 37, 196-197 (1948).

La Quinacrine, même employee aux doses fortes $(0,30$ par jour pendant deux series de cinq jours, séparées par deux jours seulement d'intervalle), ne réussit pas à guérir un quart des malades atteints de lambliase, et n'est pas dépourvue d'in-convénients immédiats.

La combinaison Quinacrine-Stovarsol a donné une réussite constante entre les mains de H., dont la posologie est la suivante: 10 à 14 jours consécutifs de traitement, en alternant un jour de Quinacrine (deux à trois comprimés par jour) avec un jour de Stovarsol (trois fois par jour 1/2 comprimé à 0.25 ).

7. Amoebiasis

Blanc, F. et Síguíer, F.: A propos de huit cas de pellagre observes au cours de dysenteries amibiennes chroniques. Bull. Mém. Soc. méd. Hop. Paris; 63, 630-636 (11 juillet 1947). 
B. et S. relatent d'abord Гobservation d'un cas de pellagre authentique sur-venant au cours d'une dysenterie amibienne, d'apparition relativement récente, mais chronique d'emblée. II ne s'agit pas d'un cas d'avitaminose d'apport, aucune déficience alimentaire n'étant signalée dans les antecedents du malade, mais bien d'une carence vitaminique par défaut d'assimilation, le malade souffrant de diarrhées depuis plusieurs mois. Trois elements permettent de dissocier ces symptômes

Referate - Abstracts - Analyses

43

entéritiques de ceux qui traduisent Tavitaminose PP: la presence des amibes dans les selles; la persistance du syndrome diarrhéique malgré Гexcellent effet de la vitamine PP sur les différents signes de pellagre; la disparition de la dysenterie sous Гeffet d'un traitement pénicilline-émétinesulfaguanidine.

C'est ainsi que $\Gamma$ attention se trouve pour la premiere fois attirée par une consequence inhabituelle des dysenteries chroniques. Dans la suite Гoccasion fut donnée aux A. d'observer plusieurs autres malades dont les principales manifestations de pellagre consistent en érythème et stomatite aphtoïde. Le traitement de vitamine PP en injections de 0,50 g par jour amène une amelioration d'une extraordinaire rapidité et d'une très grande netteté, ce qui est bien dans la note de la pellagre. Ici encore ces sujets ne souffrent d'aucune carence alimentaire, mais présentent tous une amibiase authentique, severe, chronique. Le trait commun de ces différentes dysenteries chroniques est de n'être en rien influence par la vitamine PP qui obtíent d'autre part un effet si net sur les manifestations graves de la pellagre.

Blanc, F. et Siguier, F.: Les pellagres post-dysentériques. Bull. Mém. Soc. méd. Hop. Paris; 63, 636-642 (11 juillet 1947).

La pellagre par carence d'apport en vitamine PP ne resume pas $\Gamma$ étiologie de cette curieuse affection. On réunit en effet aujourd'hui sous le terme de pellagres «secondaires» une quantité de faits étiologiques que $\Gamma$ on peut grouper ainsi sous $\Gamma$ appellation de «pellagres endogènes».

Les pellagres gastroprives sont aujourd'hui bien connues et soigneusement étudiées des points de vue clinique et experimental. On sait aussi que certaines lesions du tractus intestinal sont susceptibles de determiner d'authentiques pellagres. Ces dernières peuvent ressortir soit à un défaut d'apport entraîné par le regime nécessité par Гaffection intestinale, soit à une avitaminose secondaire aux troubles de Гab-sorption intestinale. Que se soit en Extreme-Orient ou en Europe, de nombreux médecins ont souligné les rapports unissant la pellagre et des antecedents manifestes de dysenterie chronique. Blanc et Siguier se rapportent, eux, à huit cas de dysenterie dont il leur a étê possible d'affirmer Torigine amibienne et chez lesquels ils ont pu préciser le comportement different des symptômes pellagrins et dysentériques à $\Gamma$ égard de leur traitement spécifique.

Les auteurs constatent que dans tous les cas Гérythème et la stomatite aphtoïde ainsi que les symptômes associés ne font aucun doute de par leur allure clinique et leur réponse extrêmement nette au traitement par la vitamine PP.

Les critères étiologiques de $\Gamma$ avitaminose s'écartent sensiblement des données classiques. Chez aucun des malades sauf peut-être un seul il ne fut possible de déceler un facteur carentiel exogène. Chez tous existait par contre une amibiase intestinale authentique, severe et chronique d'emblée. II s'agit souvent d'une veritable dysenterie aiguë qui tend à se perenniser mais qui, pour être difficile à enrayer, n'en n'est pas forcément irreversible.

Quant aux particularités des effets thérapeutiques les auteurs les groupent sous trois chefs: 
$1^{\circ}$ Un traitement uniquement dirigé contre la dysenterie n'améliore la pellagre qu'après un laps de temps souvent très prolongé. $2^{\circ}$ en revanche un traitement par injections d'amide nicotinique jugulant très peu de temps toute la symptomatologie pellagreuse, alors qu'elle laisse inchangé le syndrome intestinal. $3^{\circ}$ il est done plus

44

Referate - Abstracts - Analyses

prudent d'associer d'emblée le traitement anti-pellagreux à la thérapeutique anti-amibienne. Du point de vue doctrinal quelques problèmes se posent. $1^{\circ}$ On aurait tendance à établir une parenté entre pellagres endogènes post-dysentériques et sprue. Cepen-dant ces deux affections réagissent fort différemment à la thérapeutique. $2^{\circ}$ Bien que $\Gamma$ on ait recherche $\Gamma$ association d'autres avitaminoses, il ne fut pas possible par les seuls moyens cliniques de déceler une polyavitaminose B. $3^{\circ}$ Les retentissements de $\Gamma$ amibiase chronique sur les fonctions d'assimilation et d'absorption de la mu-queuse digestive sont encore mal élucidés ainsi que les déficiences vitaminiques qui peuvent en découler. II n'est pas prouvê que le deficit en vitamine PP ne relève pas d'un processus complexe où des troubles divers d'absorption et d'assimilation intesti-nales pourraient s'ajouter à une insuffisance de stockage hépatique. En outre on peut se demander si ce deficit ne joue pas de role dans la chronicité des troubles digestifs. La diarrhée fait en effet partie du tableau clinique des grandes pellagres dans les $3 / 4$ des cas. Rien n'empêche de penser qu'un veritable cercle vicieux puisse se déve-lopper, où Гamibiase, facteur d'une pellagre endogène, soit aggravée ou entretenue par les troubles digestifs lies à Tavitaminose nicotinique. Pourtant la dissociation des effets thérapeutiques des medications destinées à combattre chacun des deux syndromes est une preuve relative de leur independence.

Montel, L.: Le traitement de Гamibiase. Evolution de $\pi$ os ídées. Progrès réeents. Presse Méd.; 56, 853-854 (4 dec. 1948).

Uemetine appliquée dès 1912 dans le traitement de Гamibiase, a révolutionné la thérapeutique de cette affection terriblement chronique; mais elle n'est souvent pas suffisante pour steriliser Tintestin, et ses inconvénients - son pouvoir accumu-latif notamment - empêchent de Futiliser à fortes doses.

Les arsénícaux pentavalents, les derives de la quínoléíne sont des adjuvants précieux, et la technique de Hargreaves-Blanc (association emetine -f- pénícíllíne -j-suifaguanidíne; voir l'analyse, vol. 72, p. 262. 1947) est une méthode d'urgence remarquable, mais n'assure pas d'effets durables.

Cet article est destine à signaler un nouveau medicament, la Conessíne (spécia-lisée en France sous le nom de Roquessine), alcaloïde extrait d'une plante africaine, $\Gamma$ Holarrhena. Elle se prend per os, à raison de 5 cachets de $0,10 \mathrm{~g}$ par jour, à inter-valle de 3 heures $\Gamma$ un de $\Gamma$ autre, pendant 5 jours de suite; après quelques jovirs d'interruption, on recommence cette courte série. La Conessine agirait aussi bien dans les formes aiguës que chroniques; elle normalise rapidement les selles, calme les douleurs, et fait disparaître amibes et kystes, «au moins momentanément», reconnait $\Gamma$ A. qui ne dispose pas du recul nécessaire pour juger du pouvoir désinfec-tant définitif de cette nouvelle drogue qui, d'après M., constituerait «le plus grand progrès en matière de traitement de Гamibiase», depuis la découverte de Гémétine.

Shrapnel, B.: Oral emetine in the treatment of intestinal amebiasis. Am. ,T. Trop. Med.; 27, 527544 (1947).

Les propriétés de Гémétine rendent pénible son administration par la bouche; la voie parentérale n'est cependant pas dépourvue d'inconvénients (injections dou-ioureuses, toxicité accumulative). 
Comme, d'autre part, le chlorhydrate d'êmétine a in vitro une action amoebicide remarquable, même atix dilutions très faibles d'un

Referate - Abstracts - Analyses

45

miUionième ou d'un cinq-millionième, S. reprenant certaines tentatives a recherche si on ne pouvait pas supprimer les effets nauséeux en faisant prendre Гémétine en capsules, ne la libérant que dans la partie inférieure du tube digestif, trois ou quatre heure après Гingestion.

Les essais ont porté sur 30 malades dont 8 avaient une dysenterie aiguë, 15 une dysenterie chronique, et 7 étaient des porteurs sains de kystes; Гémétine était donnée en tablettes à 0,02 , à revêtement protecteur; le traitement fut poursuivi pendant 12 jours à la dose quotidienne de 3 prises de 2 tablettes pour les adultes; la dose totale d'émétine fut done un peu supérieure à 1,50 g.

Aucun malade ne présenta de nausées ou de vomissements et on n'observa aucun symptôme d'intoxication; ni phénomènes nerveux, ni douleurs articulaires, ni insomnie. L'acîiore du traitement fut rapide. En deux ou trois jours, la diarrhée, les épreintes et le ténesme cessèrent; les amibes disparurent des selles entre le quatrième et cinquième jour et les kystes vers le sixième jour; les ulcérations rectales amibiennes furent cicatrisées à la fin du traitement.

Les résultats furent durables; 24 malades ont été revus à distances variant de deux à neuf mois; cliniquement, la guérison était complete; la recherche des amibes et des kystes fut negative dans tous les cas sauf un.

S. n'a pas pu determiner la teneur du milieu intestinal en chlorhydrate d'émétine; il estime qu'elle a dû largement atteindre la proportion amoebicide in vitro d'un miUionième. L' $\alpha_{i}>$ sercce de tout accident toxique avec des doses supérieures à celles qu'on recommande de ne pas dêpasser par la voie parentérale, mérite d'etre soulignée.

Bliss, C. S.: L'émétine par voie buccale dans le traitement de Гamibiase intes« tinale. Am. J. Trop. Med.; 27, 527-545 (sept. 1947).

25 adultes et 5 enfants - tous amibiens - ont été traités avec succès par des comprimés de chlorhydrate d'émétine enrobée dans une substance se dissolvant seulement dans $\Gamma$ intestin. Les doses pour adultes ont été de 0,12 par jour pendant 12 jours, et pour enfants de 0,06 pendant la même période. Aucun signe toxique n'a été observe. 19 adultes et les 5 enfants traités ont été revus 2 à 9 mois après le traitement. Tous ont guéri. La substance enrobant $\Gamma$ émëtine et le medicament dissout au contact de la muqueuse intestinale fournit une concentration parasiticide suffisante pour obtenir des résultats thérapeutiques favorables.

Blanc, F. et Siguíer, F.: Sur une variété particulière d'amibiase hépatique à evolution subaiguë: les typhlo-hepatites amibiennes récidivantes à court terme.

Arch. Mai. App. Dig.; 37, 313-336 (1948).

Dans le cadre de Гamibiase hépatique, Гamibiase subaiguë se définit, selon les A., non par le caractère atténué de ses symptomes, mais par une tendance evolutive particulière se déroulant sur plusieurs mois ou années, avec poussées successives; certaines formes spéciales récidivent en moyenne tous les 45 jours environ. Cette typhlo-hépatite récidivante est rare, et survient chez d'anciens coloniaux dont la dysenterie prédomíne au niveau du caecum. Ses rechutes périodiques sans gravité quoad vitam, n'imposent pas moins un lourd handicap au malade. La lesion évolue parfois vers $\Gamma$ abcédation, d'autres guérissent après rapatriement. La thérapeutique est rendue delicate par la resistance de ces formes aux medicaments traditionnels, même associés, le court délai de la rechute empêchant de reprendre plus tôt Гémétine sans risque d'intoxication. 
Referate - Abstracts - Analyses

8. Helminthiasis

Barrelet, P.: Ua nouveau traitement taenifuge. Praxis; 37, 583-585 (1948).

Vor Jahren wurde durch Zufall entdeckt, daß das Dinitrophenol (z. B. Dinitra) ein gutes

Bandwurmmittel sei; wegen seiner Giftigkeit gelangte es nicht zur all ge-meinen Verwendung.

Ein gleicher Zufall führte zur Entdeckung der Acridinabkömm-linge als Bandwurmmittel. Die ersten Publikationen stanimen aus den Jahren 1944 und 1946 gleichzeitig aus England und Amerika. Da das Bayersche Atebrin nicht mehr erhältlich ist, wird heute das französische Atebrin unter dem Namen Quina-crine und das amerikanische Produkt Metoquina verwendet. Der Erfolg soil nach der Literatur gleich gut sein, sowohl für die Taenia wie für den Botriocephalus.

Von den vier vom Verfasser behandelten Fallen glückte die Abtreibung des Wurmes immer. Die Technik der Behandlung ist wie folgt:

Am Vortag Milchdiät.

Am Vorabend großer Einlauf, um den Dickdarm gründlich zu entleeren; bei starker Verstopfung muß der Einlauf am nächsten Morgen wiederholt werden.

Morgens nüchtern $0,8 \mathrm{~g}$ Atebrin in 8 Tabletten zu $0,1 \mathrm{~g}$ bei Bettruhe.

Vier Stunden später 30,0 g Magnesiumsulfat in $200 \mathrm{ccm}$ Wasser; wenn keine Entleerung erfolgt, Wiederholung des Abfíihrmittels nach zwei Stunden. Das Magnesiumsulfat wird dem Ricinusöl (der amerikanischen Autoren) vorgezogen, damit nicht im Falle eines Mißerfolges der Wurm durch das 01 gelöst wird und damit vermehrt Toxine zur Resorption gelangen.

Als Nebenwirkung wird gelegentlich eine leichte, flüchtige Gelbverfärbung der Skleren beobachtet, die ohne Bedeutung ist und die übrigens hauptsächlich bei prolongierter

Atebrinverabfolgung (Malariabehandlung) gesehen wird.

Der Wirkungsmechanismus des Atebrins auf den Bandwurm ist offenbar ein doppelter. Erstens wirkt das Atebrin direkt auf den Wurm. Da es außerdem durch die Galle wieder ausgeschieden wird und nach unserem heutigen Wissen die Gallen-pigmente für die Ernährung der Taenia eine wichtige Rolle spielen, ist es möglich, daß es auch auf diesem Wege toxisch und damit tödlich wirkt.

Bíyal, N.: Über die Wirksamkeit des Reinbenzins auf die Darmparasiten und seine Folgen.

Schweiz. med. Wschr.; 78, 571-572 (1948).

In zahlreichen Bezirken Nordanatoliens wird seit altersher als Volksmittel gegen Darmparasiten Petroleum und Benzin gebraucht. Nachdem der Verfasser in Selbstversuchen sich von der Harmlosigkeit und Ungiftigkeit des Reinbenzins überzeugt hatte (cave Benzin des Handels, das Methylalkohol, Blei usw. enthält), prüfte er die Wirkung dieses Volksmittels in 169 Fallen nach. Dosierung: $20 \mathrm{ccm}$ für 2-5jährige Kinder, $30 \mathrm{ccm}$ für 6-14 Jährige und $60 \mathrm{ccm}$ für Erwachsene. Um Reizsymptome von Seiten des Magens und Brennen am After zu vermeiden, wird empfohlen, das Benzin als Emulsion mit Gummi arabicum korrigiert mit Oleum Menthae piperitae zu geben.

Von 57 Ascaridenfalien hatten nur 7 ein Rezidiv, von 48 Fallen mit Taenien nur 4 und von 45 Fallen mit Oxyuren nur 8; bei letzteren werden noch Benzineinläufe ( ein Dessertlöffel auf ein Liter Wasser) während einer Woche empfohlen. Gelegentlich findet sich ein leichter

Rauschzustand, Schlafneigung, Rötung des Gesichtes und der Augen. Schwindel und Übelkeit, jedoch nie die Zeichen einer ernsten In-

Referate - Abstracts - Analyses

47 
toxikation. Das Benzin als Wurmmittel kann deshalb in der angegebenen Dosierung als erfolgreich und ohne toxische Nebenerscheinungen empfohlen werden.

Warmoes, F.: Intérêt de l'examen radiologique dans certains cas d'ascaridiose.

Acta Gastro-Enterol. Belg.; 11, 148-152 (1948).

Auf den ersten Buck scheint es abwegig zur Diagnose der Ascaridiasis die Röntgenuntersuchung heranzuziehen. Es gibt aber Ausnahmefälle, wo Wurmeier im Stuhl fehlen können (nur männliche Würmer oder sehr jugendliche Weibchen). Ferner kann so doch gelegentlich der Erfolg einer Wurmkur kontrolliert werden. Beschreibung der verschiedenen Aspekte, unter denen sich die Ascariden im Röntgenbild darstellen.

9. Tumoren

Dealon, J.: Cancer primitif du jejunum. Un cas avec survie de 14 mois. Med. J.

Austral.; 2, 771-774 (30 nov. 1946).

Les cancers primitifs du jejunum sont exceptionnels ( 0,47 à 6 p. 100 des cancers digestifs); ils paraissent frapper Thomme deux fois et demi plus souvent que la femme. Les symptômes sont des plus variables; le diagnostic n'est pose qu'à Гopé-ration ou à Гautopsie.

Le pronostic est redoutable: la mortalité opératoire est d'environ 30 p. 100 et 5 p. 100 seulement des opérés franchissent le cap de la cinquième année. L'opération comporte une entérectomie large en un ou deux temps; la reconstitution est faite selon les possibilités et le siege; lorsque la tumeur est voisine de $\Gamma$ angle duodéno-jéjunal, $\Gamma$ anastomose doit être duodéno-jéjunale. La radiothérapie est inutile et inefficace.

Lura, A.: Nuove osservazioni sul clisma opaco transbuccale del tenue. (Nouvelles observations sur le transit du grêle.) Arch. ital. mal. app. diger.; 14, 386-399 (1948).

L'A. décrit ici les résultats obtenus dans Гétude d'un cas de lymphosarcome du grêle, d'un cas de sub-occlusion et d'un cas de gastroentêro-anastomose, à Гaide de la méthode personnelle du «clisme opaque transbuccal du grêle», où Гintro-duction de la bouillie barytée se fait directement dans le duodenum par tubage.

Les avantages de cette méthode seraíent les suivants: $1^{\circ}$ Etude rapide de l'in-testin et par consequent diminution de $\Gamma$ exposition aux rayons. $2^{\circ}$ Meilleure evidence des alterations anatomiques et en particulier des infiltrations néoplasiques du grêle supérieur. $3^{\circ}$ Elimination du soupçon de subocclusxon à Гaide d'un examen rapide. $4^{\circ}$ Possibilité d'effectuer l'examen même en cas de vomissements. $5^{\circ}$ Demonstration directe du cercle vicieux dans les gastro-entéroanastomoses.

10. Sprue.

Rutishauser, E., Demole, M. et Mach, R. S.: Sprue par Iipostase coeliaque (Intestinal lipodystrophy, maladie de Whipple). Rev. Méd. Suisse Rom.; 68, 573- 580 (sept. 1948). Expose sommaire, illustré d'une photo de piece et de microphotos, d'un cas de cette maladie rare, décrite par Whipple de Baltimore en 1907.

48

Referate - Abstracts - Analyses

Elle évolue vers la mort par cachexie, après quelques annêes de diarrhées plus ou moins grasses, avec arthralgies fugaces. A Гautopsie, on trouve une énorme hypertrophie des ganglions mésentériques; au microscope, les sinus lymphatiques et la muqueuse intestinale sont infiltrés de globules graisseux, entourés de cellules géantes.

Brève discussion de la pathogenic, infectieuse ou par trouble du métabolisxne lipidique.

Stefanini, M.: Hypoproteinemia and oedema in the course of tropical Sprue.

Gastro-enterology; 11, 50-59 (July 1948). 
Of 153 Sprue patients occurring among 12500 Italian prisoners of war in India, 129 showed oedema, which seemed to be more related to the degree of anaemia than to the total serum protein level. Under treatment with diet, yeast and liver extract oedema rapidly disappeared within 7 to 10 days in 69 cases, but in 51 improvement in the anaemia as shown by the reticulocyte crisis and blood picture was accompanied by rapid extension of the oedema. This increase in oedema was related to a drop in serum protein level, which was restored to normal again in about 20 to 25 days after the beginning of treatment. The author suggests that the fall in serum proteins may be due to a demand for protein material for active erythropoiesis by the blood forming organs. It is observed also in other cases of anaemia at the start of treatment. 11. Meckelsches Divertikel

Gastich, K. J. et McNama W. L.: Cancer du diverticule de Meckel. Ann. Surg.; 124, 503-507 (sept. 1946).

Six cas d'adéno-carcinome et deux cas de carcinome médullaire du diverticule de Meckel ont été publiés jusqu'ici. C. et M. apportent un septième cas, pris au debut pour une appendicite aiguë. Opéré pour des signes de péritonite généralisée, on trouva une tumeur du grêle, que $\Gamma$ on extériorisa. La malade mourut le septième jour; à Гautopsie, on constata qu'il s'agissait d'un adéno-carcinome du diverticule de Meckel, sans envahissement des ganglions, mais avec de nombreuses métastases hëpatiques.

Voluter, G. et Thévenoz, F.: Contribution à I'étude radiologique du diverticule de Meckel. Praxis; 36, 789-792 (20. Nov. 1947).

Nach der Literatur findet man das Meckelsche Divertikel bei 2-3\% aller Indi-viduen und zwar häufiger beim männlichen Geschlecht als beim weiblichen. Es findet sich am untern Ileum zirka $45-90 \mathrm{~cm}$ von der Ileocoecalklappe entfernt und hat eine mittlere Länge von $5 \mathrm{~cm}$. Das Divertikel kann sich entzünden und eine Appendicitis vortäuschen; auch die Tuberkulose des Meckelschen Divertikels ist bekannt. Gefährlicher ist der Volvulus, der entstehen kann, wenn sich das Divertikel noch als fibröser Strang fortsetzt (Überrest des Ductus omphaloentericus). Normaler-weise, besonders wenn es nicht fixiert ist, macht das Divertikel keine Symptome. Bei Fixation an der Bauchwand kann es zu kolikartigen Schmerzen führen, eventuell auch zu Störungen desjenigen Organs, an welchem es fixiert ist (z. B. Harnblase).

Referate - Abstracts - Analyses

49

Häufiger sind schwere Bl-utungen aus dem Divertikel, seltener dagegen unstillbares und unerklärliches Erbrechen. Wenn es nicht speziell gesucht wird, gelingt es selten, das Meckelsche Divertikel radiologiscb darzustellen, da es sich nur schwer mit Kontrastbrei fiillen läßt. In gewissen Fallen sieht man nur seinen Abdruck auf einem Organ der Nachbarschaft. Gerade der vom Verfasser ausführlich beschriebene Fall zeigt diese Möglichkeit deutlich (Abdruck auf dem Colon transversum), wenn auch das erhaltene radiologische Bild vor der operativen Verifikation nicht richtig gedeutet wurde. Zur radiologischen Darstellung des Divertikels empfehlen die Verfasser eine Modifikation der Methode von Czepa, die zur Darstellung der Appendix dient. Zuerst wird der Dünndarm mit fraktionierten Breigaben gefüllt und 6-7 Stun-den p. c. werden 15-30 g Magnesiumsulfat gegeben, dann häufige radioskopische Kontrollen.

12. Ileus.

Famüíarí, R.: La polipeptidemia nella stenosi intestinale sperimentale. (La poly-peptidémie dans Tocclusion intestinale expérimentale.) Rassegna internaz. clin. terap.;28, 275-281 (1948).

La sténose expérimentale chez le lapin est toujours suivie d'une augmentation de la polypeptidémie, qui s'établit dès le premier jour de Гopération et qui, après avoir touché son 
maximum au $5 \mathrm{e}$ au $6 \mathrm{e}$ jour, redescend ensuite graduellemeat pour revenir à la normale vers le $15 \mathrm{e}$ jour.

Cette même augmentation de la polypeptidémie est évidente aussi chez les lapins témoins qui n'ont pas subi de sténose: Гon doit done en rechercher la cause dans la soudaine décharge des produits de désintégration azotée due à la destruction cellu-laire, qui se produit dans tout acte opératoire.

II existe un parallélisme evident entre la polypeptidémie, Гazotémie et le taux de Гurée urinaire, car ces trois elements augmentent et diminuent d'une façon directement proportionnelle.

L'A. croit que cela représente $\Gamma$ expression d'une defense de $\Gamma$ organisme, qui mobilise le rein et le foie afin de transformer les polypeptides en substances moins toxiques et facilement éliminables.

Brocq, P. et Eudel, F.: Les soades à lumière unique et lestées au mercure dans Гintubation du grêle et du colon. Presse Méd.; 57, 258-259 (23 mars 1949).

A la suite de deux auteurs américains, B. et E. proposent de remplacer la sonde intestinale de Miller-Abbot (terminée par un ballonnet qu'on gonfle à volonté) par une sonde à mercure: il s'agit d'un tube de caoutchouc de $5 \mathrm{~mm}$ de diamètre et $2 \mathrm{~m}$ de long (suffisant pour aller jusqu'au caecum, le grêle se plissant sur la sonde, en accordéon!), percé de 10 trous ovales dans ses 30 derniers $\mathrm{cm}$ et terminé par un sac contenant $70 \mathrm{~g}$ de mercure métallique. Le poids de ce metal entraîne rapidement et facilement la sonde jusqu'au niveau desirable, la pseudo-biloculation de Гestomac en cas de métêorisme abdominal étant la seule difficulté de son introduction.

Les A. ont realise une autre indication de la sonde à mercure (plus grosse: $10 \mathrm{~mm}$ de diamètre) pour les lavages désinfectants du colon; ils Гintroduisent alors par une boutonnière caecale, qui présente de gros avantages sur Tancienne caeco-stomie.

De magnifiques cliches (la sonde est opaque aux rayons $\mathrm{X}$ ) illustrent cet article.

Gastroenterologia. Vol. 75. Fasc. 1 (1949) 4

50

Referate - Abstracts - Analyses

Baisi, F.: La detensione intestinale mediante i moderni metodi di intubazione nel trattameato degli stati ocelusivi. (La détension intestinale par les méthodes modernes d'intubation dans le traitement des occlusions). Policl., Sez. Prat.; 55, 1212-1215 (1948).

Après avoir décrit les divers tubes employes pour la détension intestinale, $\Gamma A$. souligne les avantages du tube de Cantor. II donne, en effet, une progression intestinale plus facile et une détension meilleure. II faut le préferer à ceux de Miller-Abbott, de Johnson et de Harris.

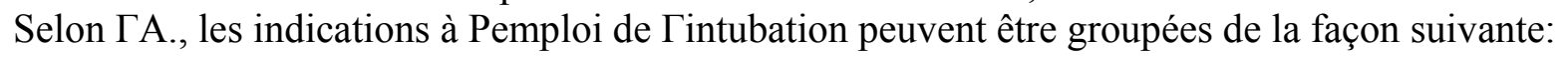
$1^{\circ}$ Ileus dynamique et dilatation intestinale des opérés; $2^{\circ}$ Ileus provoqué par un processus inflammatoire ancien, avec formation d'adhérences; $3^{\circ}$ Ileus par obstruction; dans ce cas

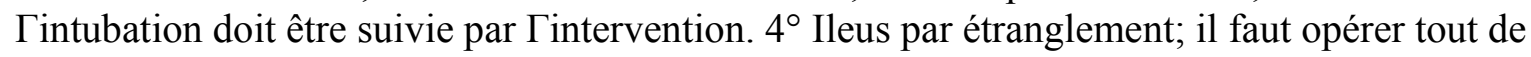
suite et intuber une fois Topé-ration achevée.

Les résultats les plus satisfaisants on été obtenus en rétablissant la continuité intestinale par la seule decompression. A ce propos $\Gamma$ A. décrit en detail un cas personnel d'ileus paralytique, dans lequel Гusage du tube de Cantor a donné un excellent résultat. Malheureusement, les résultats satisfaisants ne sont pas très frequents, mais dans la plus grande partie des cas, Гanalyse des défaillances met en evidence, à cet égard, des fautes qu'on pourrait éviter avec une technique plus soignee.

McGuff, P., Dockerty, M. B., Waugh, J. M, et Randall, L. M.: Endometriosis as cause of intestinal obstruction. Surg. Gyn. Obst.; 86, 273-288 (March 1948). 
Les dossiers de la Mayo Clinic contiennent 48 diagnostics d'endométriose intestinale entre 1920 et 1946; il s'agissait 16 fois d'une obstruction par lesion cicatri-cielle, et il faut penser à ce diagnostic en presence d'un ileus progressif et pério-dique chez une femme âgée de 30 à 50 ans, avec dysménorrhée, stérilité, et sans perte de poids. - Une constipation opiniâtre, des douleurs abdominales basses avec ballonnement existent presque toujours. La maladie peut être reconnue par la rectoscopie (masse extra-rectale sous une muqueuse ridée) ou le lavement baryté montrant une image lacunaire allongëe, inconstante, et laissant une muqueuse intacte.

Le traitement, chirurgical, ne consistera pas en resection intestinale, mais en ovariectomie bilatérale, avec ou sans colostomie temporaire.

Murado Perez, S.: Considerations étio-pathogéniques sur l'iléus aigu mécanique postappendicectomie. Rev. Españ. Enferm. Apar. Digest.; 7, 506-513 (1948).

On étudie le role joué par différents facteurs dans la production de Гiléus post-opératoire, spécialement après Гappendicectomie aiguë, tels que le drainage, la formation de péritonite plastique adhesive régionale, la non infundibilisation du moignon appendiculaire ou Tinfundibilisation très longue, la presence de coudures ou brides dans les anses iléales finales et la presence d'abscès dans le Douglas. Dans ce dernier cas Гiléus est seulement une complication de ce qui a guéri $\Gamma$ abcès.

Referate - Abstracts - Analyses

51

Stefanini, M.: Hypoproteinemia and oedema in the course of tropical Sprue. Gastro-Enterology 11, 50-59 (July 1948).

Of 153 Sprue patients occurring among 12,500 Italian prisoner of war in India, 129 showed oedema, which seemed to be more related to the degree of anaemia than to the total serum protein level. Under treatment with diet, yeast and liver extract oedema rapidly disappeared within 7 to 10 days in 69 cases, but in 51 improvement in the anaemia as shown by the reticulocyte crisis and blood picture was accompanied by rapid extension of the oedema. This increase in oedema was related to a drop in serum protein level, which was restored to normal again in about 20 to 25 days after the beginning of treatment. The author suggests that the fall in serum proteins may be due to a demand for protein material for active erythropoiesis by the blood forming organs. It is observed also in other cases of anaemia at the start of treatment. VIII. Colon, Rectum

\section{Allgemeines}

Casatí, A.: Studi suU'immagine radiologica della mucosa intestinale. Particolare aspetto della mucosa del ceco-ascendente nella sindrome addominale destra. (Etudes sur $\Gamma$ image radiologique de la muqueuse gastro-intestïnale. Aspect particulier de la muqueuse du caeco-ascendant au cours du syndrome abdominal droit.) Radiol. Med. 34, 593-607, 1948.

Employant une méthode personnelle de petit remplissage pour Гétude radiologique du gros intestin, C. a démontré dans le cæcum des sujets porteurs d'un syndrome abdominal droit, et parfois des appendicectomisés, un dessin de la muqueuse caractérisé par la presence de petits cercles tantôt voisins et minces, tantôt éloignés et plus gros. Cette image serait due à la traduction radiologique d'une tumefaction hyperplastique ou hypertrophique du système lymphatique de Гin-testin qui est liée à des conditions morbides diverses. L'A., à cet égard, souligne la fonction de «coussinet» du système lymphatique intestinal; son role d'émonctoire pour les toxines; ses rapports avec les lymphatiques du péritoine; ce qui suffirait à expliquer la reaction lymphatique de $\Gamma$ intestin. 
Kane, L. W. and G. E. Follßy: Effect of Oral Streptomycin on the Intestinal Flora. Proc. Soc. Exp. Biol. and Med. 66, 201-203, octobre 1947.

Partant du fait que la plupart des complications postopératoires lors d'inter-ventions abdominales proviennent du colibacille, du clostridium welchii ou du streptococcus fecalis, les auteurs étudient Faction de la streptomycine admimstrée par voie buccale à trois cas de côlite ulcéreuse, à deux cas préopératoires (entéros-tomie et resection) et à un individu normal. Avec la dose de 1 à $2 \mathrm{~g}$. par jour, les résultats ont été très semblables dans tous les cas. Au plus tard au deuxième jour,

52

Referate - Abstracts - Analyses

les colibacilles ont disparu des cultures de selles dans des milieux jouissant de pro-priétés antistreptomyciniques. A Гexamen direct plus aucun ge $\pi$ ne mobile ne put être décelé. Les streptocoques, les clostridies, les bacteroides et les Candida n'ont pas été affectés par la streptomycine. L'évolution des cas de côlite ulcéreuse n'a pas été influencée par la streptomycine. Les auteurs pensent par contre que cet antibiotique sera plus utile que les sulfamidés dans le traitement préopératoire des cas de ehirurgie abdominale puisque son action sur les colibacilles du colon parait certaine et que sa toxicité est très faible.

Lockwood, J. S, A. D. Young et Mc. L. Bouchelle: Appraisal of oral streptomycin as intestinal antiseptic. Ann. Surg. 129, 14-21, Jan. 1949.

Les A. se sont efforcés de résoudre le problème controversé de $\Gamma$ efficacité de la streptomycine per os, comme désinfectant intestinal, en pratiquant des numerations en series des colibacilles, streptocoques et clostridies fécaux.

Les résultats furent très irréguliers, et pratiquement nuls sur la moitié des numerations de colibacilles et des clostridies, sur les 7/8 des streptocoques. L'asso-ciation de streptomycine avec du phtalylsulfathiazol n'est pas plus efficace. II semble que le colibacille acquiert rapidement une resistance à la streptomycine, qui n'en-trave plus alors son développement.

Pemberton, H. S. et W. R. Hunter: Intestinal actinomycosis treated with streptomycin. Lancet. 256, 1094, 1949.

Les auteurs rapportent $\Gamma$ histoire d'un cas d'actinomycose intestinale qui réagit particulièrement bien, au point de vue clinique et radiologique, à la streptomycine.

Un homme, âgé de 32 ans, présentait une tumeur de la fosse iliaque droite, tumeur douloureuse à la palpation, et considêrée comme un carcinome cæcal. Une laparotomie permet de faire Tablation de cette tumeur intéressant le cæcum et la dernière anse iléale, et de terminer par une anastomose iléo-colique. L'examen anatomique rêvèle une actinomycose. Les suites opêratoires sont simples si ce n'est un petit abcès de la paroi dont le pus ne contient pas d'actinomycètes. On prescrit un traitement à la pénicilhne et aux iodures.

Deux ans plus tard apparaissent du sang et du pus dans les selles, avec du ténesme et des épreintes. Une rectoscopie montre une rnuqueuse rectale cedématiée et re-couverte de pus contenant des filaments mycéliens. Un transit montre une forte diminution de calibre de $\Gamma$ anse grêle anastomosée au colon transverse, et on palpe de nouveau une tumefaction sensible correspondant à cette region. La streptomycine est alors administrée à la dose de $1 \mathrm{~g} . / \mathrm{j}$. per os durant 30 jours. Les signes cliniques et rectoscopiques disparaissent en 10 jours, alors qu'un transit pratique un mois plus tard montre une anse grêle de calibre normal laissant passer rapidement le repas baryté.

Trois autres auteurs ont rapporté des cas d'actinomycose traités avec succès par la streptomycine, un cas d'atteinte de la mâchoire, un cas de méningite, et un cas de lesion respiratoire. Notons 
cependant que tous les types d'actinomyces ne sont pas sensibles à la streptomycine, et que les cas traités avec succès étaient certaine-ment infectés par un type sensible. Les résultats obtenus justifient pourtant $\Gamma$ emploi de la streptomycine.

Referate - Abstracts - Analyses

53

Posey, E. L., H. S. Brown et J. A. Bargen: The response of human intestinal motility to tetraethyl ammonium chloride. Gastro-Enterology 11, 83-90, July 1948.

Tetraethyl Ammonium Chloride (TEAC) was given to 4 volunteers in a dosage varying from 200 to $400 \mathrm{mg}$. injected intravenously. All the subjects had colostomy openings and a balloon connected to a water reservoir and recording apparatus was inserted in the portion of colon proximal to the opening.

In all four subjects TEAC rapidly inhibited the motor activity of the intestines, but the degree varied in each individual. In general the period of inhibition was of longer duration than the similar inhibition of gastric motility, and varied between 30 minutes and 65 minutes. The tone of the intestine was also depressed in 3 of the 4 subjects.

Tenner, R, J.: Lipomas of large and small intestine. Journal-Lancet, Minneapolis, 68, 12-23, Jan. 1948.

Analyse de 12 observations de lipomes de Гintestin (surtout du colon), produisant des symptômes assez sérieux pour nécessiter une intervention chirurgicale: il s'agissait avant tout de douleurs, souvent compliquées de vomissements. La perte de poids était habituelle, que Гintestin fonctionne en diarrhée ou en constipation. On trouve constamment une ulcération de la muqueuse recouvrant le lipome, due soit à un processus d'invagination partielle, soit au traumatisme en cas de retrostruc-tion; soit à un infarctus vasculaire consécutif au développement de la tumeur; malgré cefte perte de substance, il y avait rarement du sang dans les selles.

Le diagnostic pose avant $\Gamma$ opération était, la plupart du temps, celui de tumeur maligne, étant

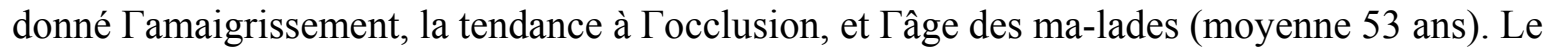
traitement chirurgical consista tantôt en resection simple de la lesion, avec ou sans entéroanastomose, ou de resection du segment d'implantation de la tumeur.

Rebell, F. G.: The problem of foreign bodies in the colon and rectum. Am. J. Surg. 76, 678-686, 1948.

Description de 24 cas de corps étrangers du rectum et revue de cette question. Les corps étrangers proviennent de:

$1^{\circ}$ Deglutition (cure-dents, aretes de poisson, morceaux d'os, pieces de prothèses, aiguilles) qui, ayant parcouru tout $\Gamma$ intestin, s'arrêtent dans une crypte rectale où ils donnent généralement naissance à une fistule.

$2^{\circ}$ Oublis dans $\Gamma$ abdomen lors d'une intervention (compresses, tampons ou drains), qui s'éliminent par le sigmoïde après avoir perforê sa paroi.

$3^{\circ}$ Perte dans le rectum par faute technique (thermomètres, canules rectales, dilatateurs), ou introduits par perversion (de la carotte à la bouteille de bière).

Les regies qui doivent guider le chirurgien dans le diagnostic et dans $\Gamma$ extraction sont variables et dans certains cas Гopération par voie abdominale est inevitable. L'anus de derivation est indiqué dans les cas où il existe des blessures importantes de la paroi rectale ou de $\Gamma$ infection. La rupture de $\Gamma$ intestin peut survenir spontanément ou à la suite des manoeuvres d'extraction; elle commandera la laparotomie d'urgence.

54

Referate - Abstracts - Analyses 


\section{Colitis chronica}

Famed, P.: Le acque salsoiodiche nella terapia delle dispepsie e delle côliti croniche (Les eaux iodo-chlorurées dans le traitement des dyspepsies et des colites chro-niques). Arch. ital. mal. app. diger. 14, 337-343, 1948.

Les eaux iodo-chlorurées (de Bagni delle Ponetta, Appennin) exercent une action excitante qui entraîne une amelioration des dyspepsies hyposthéniques et le retour à $\Gamma$ état normal du fonctionnement gastrique et hépatique. Au contraire elles sont tout à fait contrindiquées dans les dyspepsies hypersthéniques, surtout préulcé-reuses, car elles entraînent alors une augmentation des souiFrances. Introduites par lavement, elles donnent des succès rapides dans les reliquats de Tamibiase intestinale, dans la côlite muco-membraneuse, dans la constipation soit atonique soit spastique, ainsi que dans la dyschésie et dans les troubles à distance du colon dus à des maladies de Tappareil genital féminin.

La balnéo-thérapie exerce une action bienfaisante sur le système neuro-végétatif.

Crohn, B. B., J. H. Garlock et H. Yamis: Right-sided (regional) colitis. J. A. M. A.

134, 334-338, 24 mai 1947.

La côlite ulcéreuse idiopathique est localisée, 8 fois sur 100, au cæco-ascendant, avec d'ailleurs tendance à Tenvahissement de tout le cadre côlique; sa frequence est maximale entre 20 et 30 ans, avec predominance dans le sexe féminin.

La côlite ulcéreuse droite est moins grave que les autres formes de la maladie; elle se manifeste par des diarrhées moins profuses et moins répêtées, rarement sanglantes, et précédées de coliques; parmi les complications locales, les fistules -surtout péri-anales - sont communes, Mais Гétat general est touché: temperature irrégulière, et souvent élevée; leucocytose; participations des articulations (rhuma-tisme dysentérique), des yeux (iritis, kératite, conjonetivite phlycténulaire), du cæur (endocardite valvulaire).

Le pronostic de ce syndrome n'est pas mauvais: il y a 84\% de survivants dans la série des A. Le traitement consiste en longues périodes de Succinyl- ou phtalyl-sulphatiazol, additionnés d'extrait de foie en injections. En cas d'échec, on discutera Гinter-vention chirurgicale, après s'ètre assure de Гintégrité de la region recto-sigmoïdienne. Elle consiste généralement à pratiquer d'abord une iléostomie, suivie dans un second temps (3 à 6 mois plus tard) d'une colectomie subtotale, et terminée, si possible, par le rétablissement de la continuité iléosigmoïdienne. Sur 46 malades opérês, deux ont succombé rapidement, les 43 autres se portent bien et out repris leur acti-vité professionnelle, malgré 3 à 5 selles molles par jour.

Sterne, J.: Côlites d'origine histaminique. Gaz. méd. France 55, 615-616, sep-tembre 1948. L'auteur isole une forme particulière de côlite rare, caractérisée par Гexistence de troubles intestinaux à poussées avec incidents mineurs entre les attaques, indé-pendants de toute infection ou parasitisme, accompagnés parfois de troubles aller-giques, immédiatement supprimês par les antihistaminiques de synthèse et dont $\Gamma$ origine allergique peut être admise. 